\title{
Herbert Obinger
}

\section{Die Finanzkrise und die Zukunft des Wohlfahrtsstaates}

\section{Einleitung ${ }^{1}$}

Die globale Finanzkrise führte in vielen entwickelten Demokratien zur schwersten Wirtschaftskrise seit den 1930er Jahren. Ohne Zweifel hat der Wohlfahrtsstaat kurzfristig wesentlich dazu beigetragen, dass die sozialen Verwerfungen der jüngsten Rezession bisher weit weniger verheerend waren als jene der Großen Depression. $^{2}$ Von dieser kurzfristigen Erfolgsbilanz des Sozialstaates sind aber die mittelund langfristigen Krisenfolgen zu unterscheiden. Die These dieses Beitrags lautet, dass die Bürger der fortgeschrittenen Wohlfahrtsstaaten krisenbedingt auf lange Sicht mit erheblichen Einschnitten in die sozialen Sicherungssysteme zu rechnen haben. Meine Argumentation setzt sich aus vier Bausteinen zusammen, die auch die ersten Abschnitte dieses Beitrags strukturieren. Hauptursache für die zu erwartende Phase restriktiver Sozialpolitik ist die Schuldenkrise, die sich in vielen Ländern im Zuge der Wirtschaftskrise dramatisch zugespitzt hat. Künftig wird der Wohlfahrtsstaat aus drei Gründen stärker als bislang in das Zentrum von Einsparungsbemühungen geraten: Erstens ist heute in der OECD-Welt im Schnitt über die Hälfte aller öffentlichen Ausgaben im Sozialbereich gebunden. Anders als in der Vergangenheit kann zweitens der Einsparungsdruck künftig nicht mehr so leicht in nicht-sozialstaatliche Politikfelder umgelenkt werden. Hier wurde nämlich bereits in den letzten beiden Jahrzehnten der Rotstift in einem Ausmaß angesetzt, ${ }^{3}$ dass mittlerweile kaum noch umfangreiche Ausgabenkürzungen zu erreichen sind, ohne zentrale Funktionen des Staates zu gefährden. Drittens hat sich schließlich der Handlungsspielraum des Nationalstaates auf der Einnahmenseite verringert. Der Umstand, dass die Fiskalkrise Kürzungen in der Sozialpolitik wahrscheinlicher machen wird, bleibt wiederum für Politik und Demokratie nicht folgenlos. Dieser Aspekt wird im letzten Abschnitt dieses Beitrags thematisiert.

Die in diesem Beitrag diskutierte Frage nach den Auswirkungen der jüngsten Wirtschaftskrise auf die Zukunft des Wohlfahrtsstaates ist untypisch für die Poli-

1 Die Ereignisse bis zur Drucklegung haben die Prognosen dieses Aufsatzes weitgehend bestätigt. Zahlreiche Länder haben seitdem einen Austeritätskurs forciert, der insbesondere in hochverschuldeten Staaten drastische Kürzungen von Sozialleistungen beinhaltet. Ich danke Wolfgang Streeck, Carina Schmitt, Oliver Pamp, Reinhard Blomert und den anonymen Gutachtern für hilfreiche Hinweise.

2 Kaasch et al. 2010; Vis et al. 2011.

3 Castles 2007.

Leviathan, 40. Jg., 3/2012, S. 441- 461 
tikfeldanalyse, die üblicherweise mit rückblickenden Hypothesen arbeitet ${ }^{4}$ und nur selten Zukunftsprognosen abgibt. Angesichts dessen, was auf dem Spiel steht, soll der zugegebenermaßen spekulative Versuch dennoch gewagt werden. Der Ausblick in die Zukunft beruht auf einer Zusammenschau von Verschuldungsdaten und der Entwicklung politikfeldspezifischer Staatsausgaben in 21 OECD-Kernländern ${ }^{5}$ im Zeitraum von 1990 bis zum Vorabend der Finanzkrise.

\section{Von der Finanzmarkt- zur Fiskalkrise}

Die zur Abfederung der wirtschaftlichen Krisenfolgen aufgelegten Bankenrettungsund Konjunkturprogramme sowie die rezessionsbedingten Steuerausfälle und Ausgabensteigerungen im Sozialbereich ${ }^{6}$ haben in fast allen entwickelten Demokratien die Staatsverschuldung binnen weniger Jahre massiv in die Höhe getrieben. ${ }^{7}$ Länder wie Großbritannien verzeichneten das höchste Budgetdefizit seit Kriegszeiten, und auch in anderen Ländern hat die Staatsverschuldung Rekordhöhen erreicht. In Deutschland sind nach vorläufigen Angaben des Statistischen Bundesamts allein im Jahr 2010 die Staatsschulden um mehr als 304 Mrd. Euro angewachsen, sodass am Jahresende der Schuldenstand annähernd die Zwei-Billionen-Euro-Marke erreicht hat. ${ }^{8}$ Abbildung 1 zeigt für 21 langjährige Mitgliedsländer der OECD die Bruttostaatsschulden in Prozent des Bruttoinlandsprodukts (BIP) im Jahr 2007 (schwarzer Balken) sowie die laut Prognose der OECD im Zuge der Finanzkrise bis 2011 angehäuften Schulden (grauer Balken). Mit Ausnahme von Schweden, Norwegen und der Schweiz sind die Staatsschulden seit Beginn der Krise geradezu explodiert (grauer Balken). Fast alle Euro-Länder ${ }^{9}$ werden 2011 die 60-Prozent-Verschuldungsgrenze überschreiten. Demgegenüber bleibt die Schuldenquote in den Antipoden, der Schweiz sowie den drei nordischen Nicht-Euro-Ländern unterhalb von 60 Prozent des Bruttoinlandsprodukts.

Dieser dramatische Anstieg der Staatsverschuldung im Zuge der jüngsten Wirtschaftskrise hat die in einigen Ländern in den 1990er Jahren erzielten Haushaltskonsolidierungserfolge ${ }^{10}$ binnen kurzer Zeit wieder zunichte gemacht. Für das Jahr 2011 wurde für sechs der 21 hier betrachteten Länder eine Verschuldungsquote von

4 Scharpf 2000 a, S. $56 \mathrm{ff}$.

5 Australien, Belgien, Dänemark, Deutschland, Finnland, Frankreich, Griechenland, Irland, Italien, Japan, Kanada, Neuseeland, Niederlande, Norwegen, Österreich, Portugal, Spanien, Schweden, Schweiz, Vereinigtes Königreich und USA.

6 Adema et al. 2011.

7 Wagschal, Jäkel 2010; OECD 2011, S. 254.

8 Siehe http://www.staatsverschuldung.de/destatis2011-02-21.pdf (Zugriff vom 10.03.2011). Dieser Rekordzuwachs ist wesentlich der Einrichtung von »Bad Banks« geschuldet.

9 Die in diesem Beitrag nicht berücksichtigten EU-Kleinstaaten Luxemburg und Estland bilden eine Ausnahme.

10 Wenzelburger 2010, S. 39. 
über 100 Prozent des BIP prognostiziert. Vor allem im Euroraum sind künftig umfangreiche Konsolidierungsanstrengungen unumgänglich. Ähnliches gilt aber auch für wirtschaftliche Supermächte wie Japan und die Vereinigten Staaten, wobei für Japan die finanziellen Konsequenzen der Tsunami- bzw. Atomkatastrophe in diesen Zahlen noch gar nicht berücksichtigt sind. ${ }^{11}$ Fiskalpolitische Austerität wird daher für lange Zeit die politische Agenda in weiten Teilen der OECD-Welt bestimmen - mit beträchtlichen Konsequenzen für die Demokratie. ${ }^{12}$

Abbildung 1: Staatsverschuldung in Prozent des BIP 2007 (schwarzer Balken) und Veränderung der Staatsverschuldung zwischen 2007 und 2011 (grauer Balken)

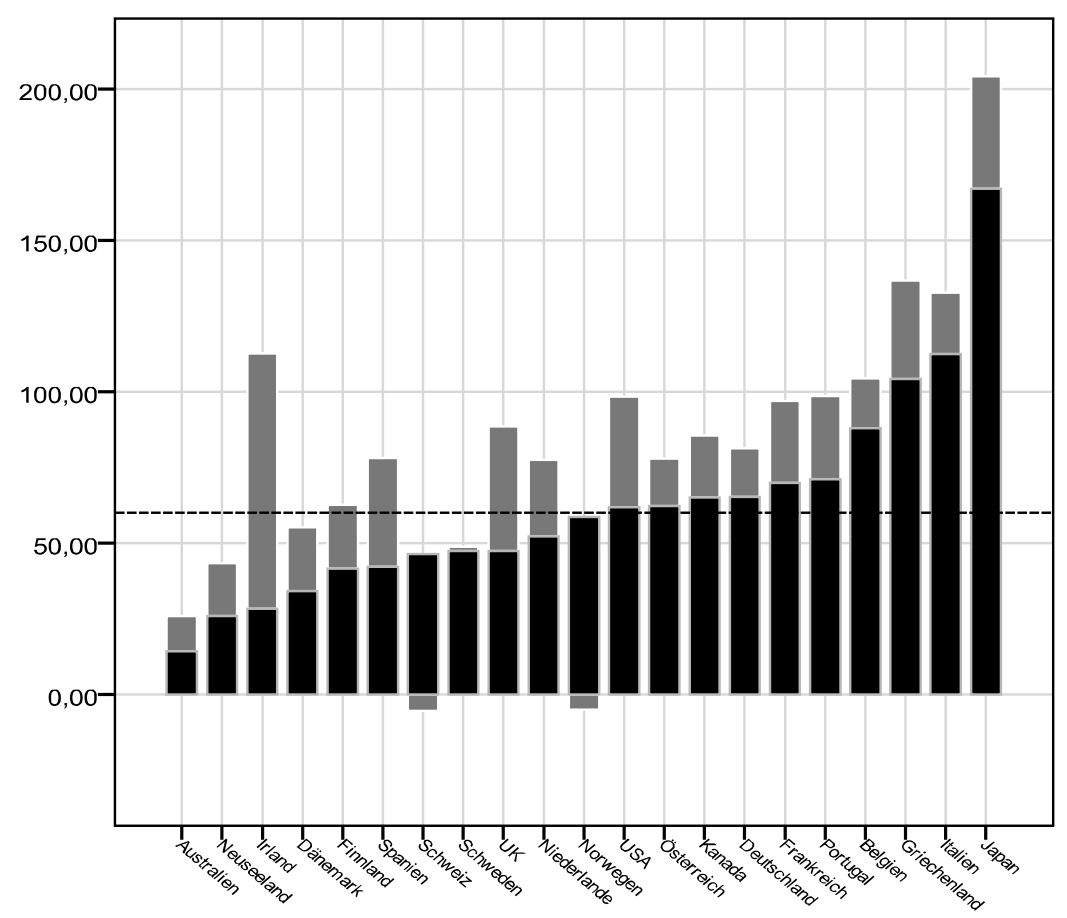

Datenbasis: OECD 2010 a; dargestellt ist die Bruttoschuldenquote.

Grundsätzlich stehen mit Ausgabenkürzungen oder Einnahmenerhöhungen nur zwei Wege zur Haushaltssanierung offen. Beide Optionen sind jedoch unpopulär

11 Allein die Folgekosten der Tsunamikatastrophe werden auf 217 Mrd. Euro geschätzt (Frankfurter Allgemeine Zeitung, 24. März 2011, S. 11). Eine japanische Besonderheit ist jedoch die extrem hohe Inlandsverschuldung.

12 Streeck, Mertens 2010. 
und in demokratisch verfassten Systemen für die politischen Eliten mit Risiken verbunden. Dies gilt insbesondere für Einsparungsversuche bei den sozialen Sicherungssystemen des Wohlfahrtsstaates, der aufgrund seiner Popularität und seiner zahlenmäßig großen Unterstützerbasis als besonders gut geschützt vor drastischen Einschnitten gilt. ${ }^{13}$

Mein Argument lautet nun, dass der Sozialstaat künftig erheblich stärker unter Einsparungsdruck geraten wird, als das bislang der Fall war. ${ }^{14}$ Dies hängt mit drei eng miteinander verknüpften Faktoren zusammen, die in den nächsten Abschnitten - allerdings getrennt voneinander - diskutiert werden.

\section{Der Wohlfahrtsstaat als Kürzungsobjekt: Das »Sutton'sche Gesetz«}

Die erste Erklärung ist denkbar einfach und lässt sich mit einer Anekdote illustrieren. Der amerikanische Bankräuber Willie Sutton (1901-1980) soll angeblich auf die Frage eines Reporters, warum er eine Bank überfallen habe, schlicht geantwortet haben: »Because that's where the money is « ${ }^{15}$ Das »Sutton'sche Gesetz « lehrt, den Blick auf das Naheliegende zu richten. Es ist das schiere fiskalische Gewicht des Wohlfahrtsstaates, welches ungeachtet der institutionellen Vielfalt wohlfahrtsstaatlicher Arrangements die Begehrlichkeiten der Haushaltspolitiker weckt. Der Wohlfahrtsstaat ist heute überall mit Abstand der größte Ausgabenposten der öffentlichen Hand. In den 21 OECD-Ländern betrug im Jahr 2007 der Anteil der öffentlichen Sozialausgaben an den gesamten Staatsausgaben im Durchschnitt 50,4 Prozent. Die nationalen Unterschiede sind dabei relativ gering (Abbildung 2). Der Anteil der Sozialausgaben an den gesamten öffentlichen Ausgaben reichte von 42,7 Prozent in Kanada bis zu 57,8 Prozent in Deutschland, wo der Sozialstaat andere Politikfelder fiskalisch offensichtlich besonders stark an den Rand drängt. ${ }^{16}$

Am Vorabend der Finanzkrise entfiel daher rund eine Hälfte der gesamten Staatsausgaben auf den Wohlfahrtsstaat, während die andere für die restlichen Politikfelder reserviert war. ${ }^{17}$ Wichtig für die weitere Argumentation ist der Umstand, dass das relative Gewicht des Sozialstaates - also der Anteil der Sozialausgaben an den öffentlichen Gesamtausgaben - in den letzten drei Jahrzehnten in allen Ländern deutlich zugenommen hat. 1980 waren im Durchschnitt lediglich 38 Prozent der gesamten öffentlichen Ausgaben im Sozialbereich gebunden, und dementsprechend entfielen auf alle nicht-sozialstaatlichen Politikfelder 62 Prozent der Gesamtausgaben. Diese Relation hat sich in einigen Ländern, darunter auch Deutschland, stark zugunsten der Sozialausgaben verändert. Hinzu kommt, dass das fiskalische Gewicht des Sozialstaates im Zuge der Wirtschaftskrise weiter zugenommen hat.

13 Pierson 1994, 1996; Svallfors 2010.

14 Vgl. für Deutschland Streeck 2009.

15 Willie Sutton hat später in einem Buch diesen Satz bestritten. Ich danke Francis G. Castles für den Hinweis auf diese Anekdote.

16 Vgl. Schmidt 2005, S. 291.

17 Vgl. Castles 2007. 
Allein zwischen 2007 und 2010 ist im OECD-Durchschnitt die öffentliche Sozialausgabenquote um drei Prozentpunkte angestiegen. ${ }^{18}$ Wenn daher eine Regierung zu Haushaltsdisziplin und Einsparungen gezwungen ist, führt in Zukunft wohl kaum noch ein Weg an Ausgabenkürzungen bei den sozialen Sicherungssystemen vorbei.

Abbildung 2: Anteil der öffentlichen Sozialausgaben in Prozent der gesamten Staatsausgaben (2007)

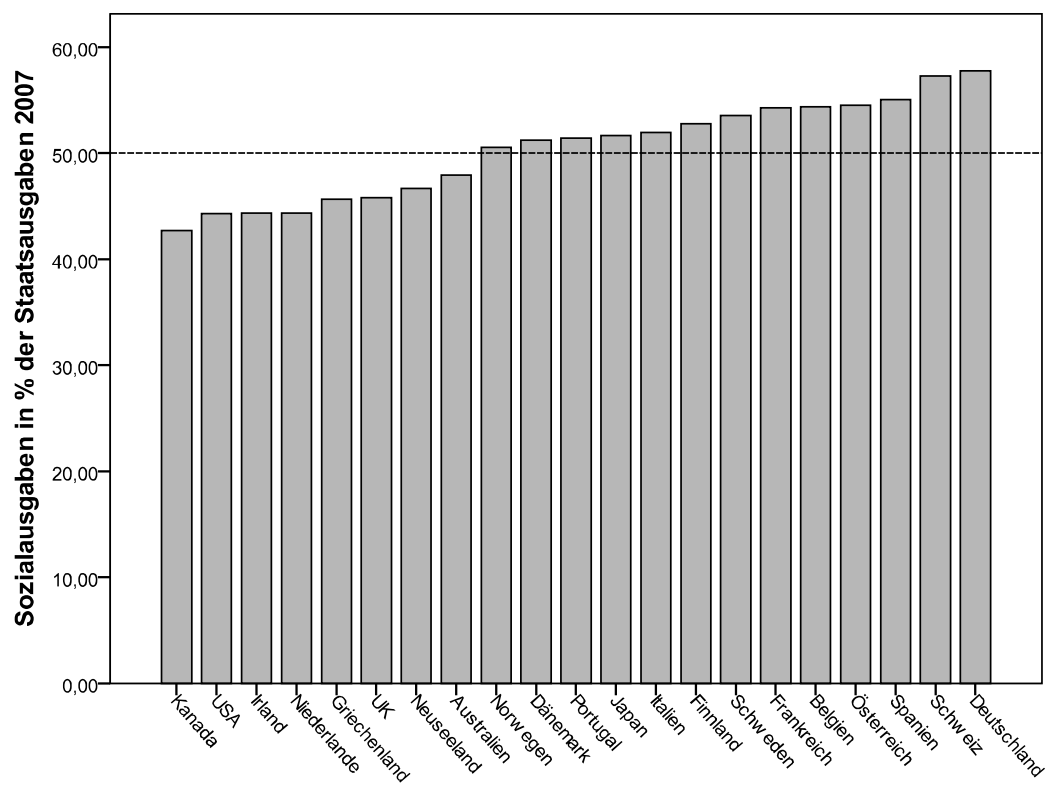

Quelle: OECD 2010 b.

Abbildung 3 zeigt jene Länder, in denen dies besonders wahrscheinlich ist. Dargestellt sind der Anteil der Sozialausgaben an den Gesamtausgaben der öffentlichen Hand (Ordinate), die für 2011 prognostizierte Verschuldungsquote (Abszisse) sowie - als horizontale und vertikale Linien - die jeweiligen Mittelwerte. Kürzungen im Sozialbereich sind insbesondere in den im rechten oberen Quadranten gruppierten Ländern wahrscheinlich. Gegenteiliges sollte für die Länder im linken unteren Quadranten gelten. Dort ist nämlich nicht nur die Haushaltssituation weitaus weniger angespannt, sondern auch das relative fiskalische Gewicht des Sozialstaates ist deutlich niedriger, sodass Einsparungen in nicht-sozialstaatlichen Politikfeldern leichter zu erzielen sind. Hinzu kommt ein geringerer Druck auf die Sozialausgaben aufgrund eines vergleichsweise günstigen demografischen Profils.

18 Adema et al. 2011, S. 10.

Leviathan, 40. Jg., 3/2012 
Abbildung 3: Staatsverschuldung und das relative Gewicht des Wohlfahrtsstaates

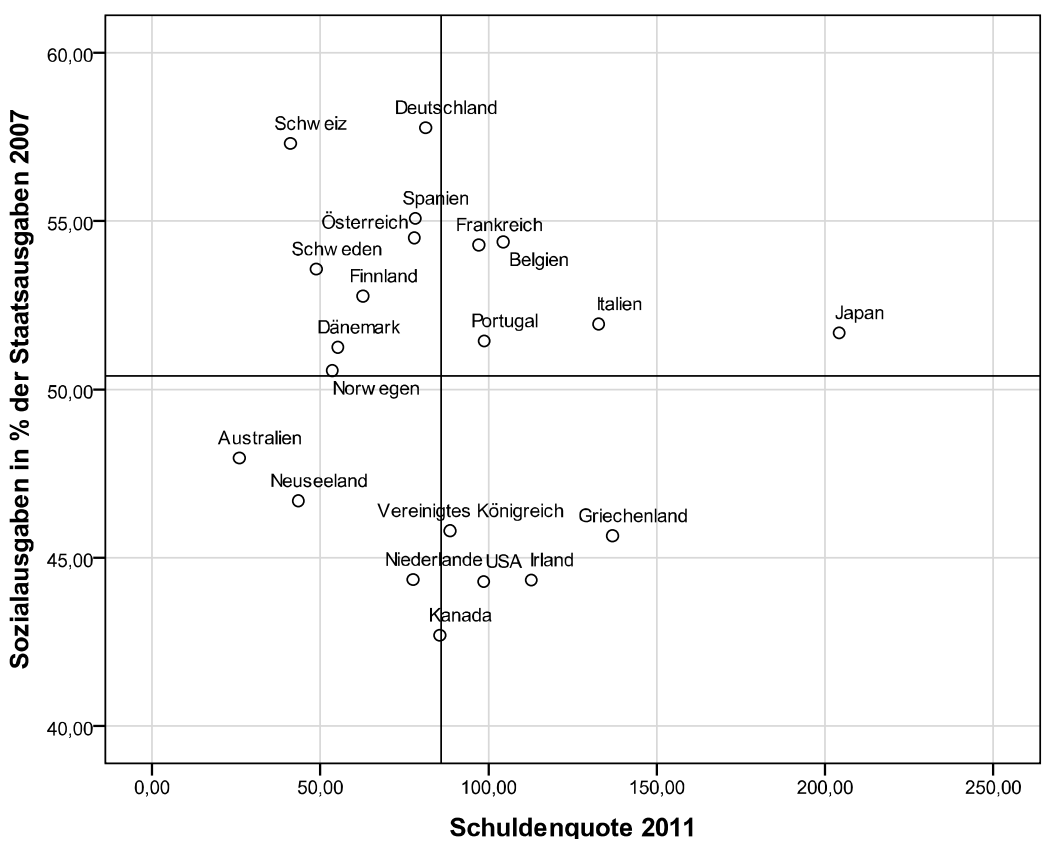

Datenbasis: OECD 2010 a, 2010 b.

Anmerkung: horizontale Linie: Mittelwert Sozialausgaben; vertikale Linie: Mittelwert Staatsverschuldung

Eine hohe Staatsverschuldung und der damit verbundene hohe Budgetkonsolidierungsdruck sind jedoch keine neuen Phänomene. ${ }^{19}$ Bereits in den 1990er Jahren hatte eine Reihe von OECD-Ländern mit hoher Staatsverschuldung zu kämpfen, und die Maastrichter Konvergenzkriterien zwangen insbesondere die europäischen Länder zu einer restriktiven Haushaltspolitik. Einige Staaten war in ihren Konsolidierungsbemühungen durchaus erfolgreich. ${ }^{20}$ Angesichts der Tatsache, dass die Sozialausgaben seit 1980 in fast allen Ländern gestiegen sind, ${ }^{21}$ muss geklärt werden, warum erstens der Wohlfahrtsstaat weiter gewachsen bzw. von umfangreichen Einschnitten relativ verschont blieb und warum zweitens künftig im Sozialbereich mit stärken Kürzungen zu rechnen ist.

19 Reinhart, Rogoff 2009.

20 Zohlnhöfer 2009.

21 OECD $2010 \mathrm{~b}$. 


\section{Das Ende der New Politics of the Welfare State: Time Matters}

Zur Beantwortung dieser Fragen stütze ich mich weitgehend auf Arbeiten von Paul Pierson, betone dabei aber, dass die von ihm als Hauptursache für die Robustheit des Wohlfahrtsstaates identifizierte neue Politiklogik (New Politics of the Welfare State) kontextabhängig ist und Zeit eine wichtige Rolle spielt. Das Argument lautet verkürzt, dass diese strategische Option nur unter spezifischen Voraussetzungen zur Verfügung stand und mittlerweile weitgehend ausgeschöpft ist.

Piersons These, wonach der Wohlfahrtsstaat als Folge einer neuen und auf der Angst vor Schuldzuweisungen (blame avoidance) basierenden Politiklogik immun gegenüber umfassenden Rückbauversuchen ist, ${ }^{22}$ findet für die meisten OECD-Länder für das letzte Viertel des 20. Jahrhunderts empirische Bestätigung. Das heißt nicht, dass keine Kürzungen im Sozialbereich vorgenommen wurden, aber im Vergleich zu anderen Politikbereichen kam die Sozialpolitik relativ glimpflich davon. Hauptgründe für die Resistenz des Wohlfahrtsstaates gegenüber radikalem Rückbau sind demnach seine hohe Popularität und die breite Unterstützung der Sozialpolitik durch die Leistungsbezieher und Beschäftigten des Wohlfahrtsstaates, die zusammen einen erheblichen Anteil der (wahlberechtigten) Bevölkerung repräsentieren. Politiker, die Kürzungen von Sozialleistungen anstreben, sehen sich daher einem hohen politischen Abwahlrisiko ausgesetzt. Einschnitte in das soziale Netz verursachen nämlich kurzfristig bei den Betroffenen konzentrierte und schmerzhafte Kosten (z.B. geringeres Transfereinkommen), stiften aber langfristig lediglich einen diffusen Nutzen (z.B. höheres Wirtschaftswachstum), von dem der einzelne Bürger nicht notwendigerweise profitieren wird. Da vor allem die von restriktiver Sozialpolitik unmittelbar und kurzfristig Betroffenen politisch gegen Leistungseinschränkungen mobil machen, verzichten wiederwahlorientierte Politiker entweder auf drakonische Kürzungen oder sie versuchen, die Sichtbarkeit unpopulärer Maßnahmen zu kaschieren. Zwar sind Leistungskürzungen deshalb nicht ausgeschlossen, in jedem Fall ist aber nicht mit radikalen Einschnitten zu rechnen.

Piersons Thesen erfuhren neben viel Lob zum Teil aber auch heftigen Widerspruch. Während Korpi und Palme ${ }^{23}$ zum Teil erhebliche Leistungseinschnitte bei den Lohnersatzraten nachweisen konnten, wiesen andere Autoren zu Recht auf einen tiefgreifenden Umbau der sozialen Sicherungssysteme (z.B. aktivierende Arbeitsmarktpolitik, Teilprivatisierung der Alterssicherung, Ausbau der Familienpolitik) seit den 1990er Jahren hin. ${ }^{24}$

Paul Pierson reagierte lange Zeit nicht auf diese Kritik. Umso interessanter ist ein aktueller Beitrag, in dem er sich mit der Entwicklung des Wohlfahrtsstaates in langfristiger Perspektive auseinandersetzt und nachdrücklich seine Thesen bekräftigt. ${ }^{25}$ Trotz fundamentaler Veränderungen in Politik, Wirtschaft und Gesellschaft seit Mitte der 1970er Jahre zeichne sich der Wohlfahrtsstaat durch ein »surprising

22 Pierson 1994, 1996.

23 Korpi, Palme 2003.

24 Vgl. z.B. Gilbert 2004; Hemerijck 2011.

25 Pierson 2011.

Leviathan, 40. Jg., 3/2012 
level of stability « aus. Und weiter: "Natürlich gibt es signifikante Veränderungen über vier Dekaden - das ist völlig klar. Aber gemessen an zentralen Kriterien wie dem Inklusionsgrad und den Lohnersatzraten sind die meisten Sozialstaatsprogramme in den reichsten Demokratien ungefähr so generös wie sie immer waren. Trotz dramatischer sozialer Veränderungen und großen fiskalischen Drucks in der Vergangenheit ist die überwältigende Mehrheit der Programme heute generöser als gegen Ende des so genannten Goldenen Zeitalters. Kurz, das allgemeine Ausmaß an Einschnitten und das Muster der Variation zwischen den Programmen passen zu einer theoretischen Perspektive, die betont, dass mit der Expansion des Sozialstaates neue Interessengruppen entstanden sind, die die Basis politischer Unterstützung für den Sozialstaat verbreitern « ${ }^{26}$ Pierson räumt allerdings ein, dass dieser Befund ${ }^{27}$ nicht zwangsläufig für die Zukunft gelten muss: »Vielleicht beginnt das Zeitalter der wirklichen Einschnitte erst jetzt ${ }^{28}$

Genau dies wird in diesem Beitrag behauptet: Die Konsolidierung der Staatsfinanzen ist zwar keine neue Herausforderung, und sie ist auch alles andere als unmöglich, sie kann künftig jedoch kaum noch unter Ausklammerung des Sozialstaates erreicht werden. Eine Ursache wurde mit dem wachsenden fiskalischen Gewicht des Sozialstaates bereits ausgemacht. Als weiterer Grund kommt hinzu, dass bestimmte in der Vergangenheit praktizierte Wege der Haushaltskonsolidierung künftig nicht mehr oder nur noch eingeschränkt beschritten werden können.

Eine Rückschau in die 1990er Jahre zeigt, dass eine Reihe von Ländern bereits damals aufgrund des kontinuierlichen Anstiegs der Staatsverschuldung seit den beiden Ölpreisschocks mit einem hohen Konsolidierungsbedarf konfrontiert war. ${ }^{29}$ Die damit verbundenen hohen Zins- und Tilgungslasten (durch hohe Zinssätze in den 1980er Jahren), die Abflachung des Wirtschaftswachstums, steigende Arbeitslosigkeit sowie - in den 1990er Jahren - die Maastrichter Konvergenzkriterien haben den Spardruck weiter erhöht. Tatsächlich konnten in den 1990er Jahren viele Länder ihre Staatsausgaben drosseln. Wie Abbildung 4 anhand von Boxplots zeigt, ist zwischen 1990 und 2000 die Staatsquote erstmals seit dem Zweiten Weltkrieg wieder gesunken. Der Median sank von 48,3 Prozent auf 42,3 Prozent. Zwischen 2000 und 2007 ist die Staatsquote zwar wieder leicht angestiegen, sie blieb im Mittel jedoch deutlich unter dem Niveau des Jahres 1990.

26 Pierson 2011, S. 18, 20.

27 Für eine Kritik siehe Hemerijck 2011.

28 Pierson 2011, S. 21.

29 Wagschal, Wenzelburger 2008, S. 12. 
Abbildung 4: Die Staatsquote in 21 OECD-Ländern seit 1990

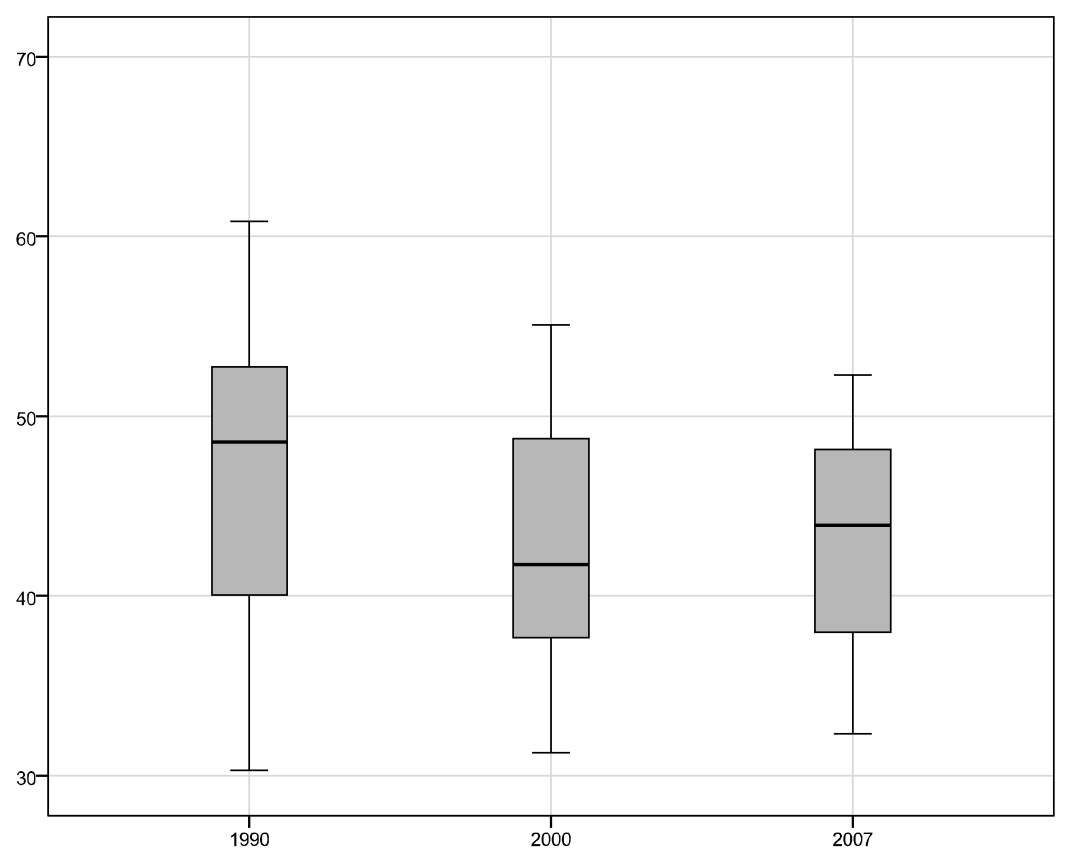

Datenbasis: OECD 2010 a.

Demgegenüber blieb der Median der Sozialausgaben im Verhältnis zum Bruttoinlandsprodukt in den 1990er Jahren praktisch unverändert (Abbildung 5), der arithmetische Mittelwert ist sogar von 19,7 Prozent (1990) auf 21,0 Prozent im Jahr 2000 angestiegen. Dieser ansteigende Trend setzte sich in den 2000er Jahren weiter fort. Zwischen 1980 und 2007 ist die Sozialausgabenquote in den 21 Ländern im Schnitt sogar um über vier Prozentpunkte angewachsen. 
Abbildung 5: Die Sozialausgabenquote in 21 OECD-Ländern seit 1990

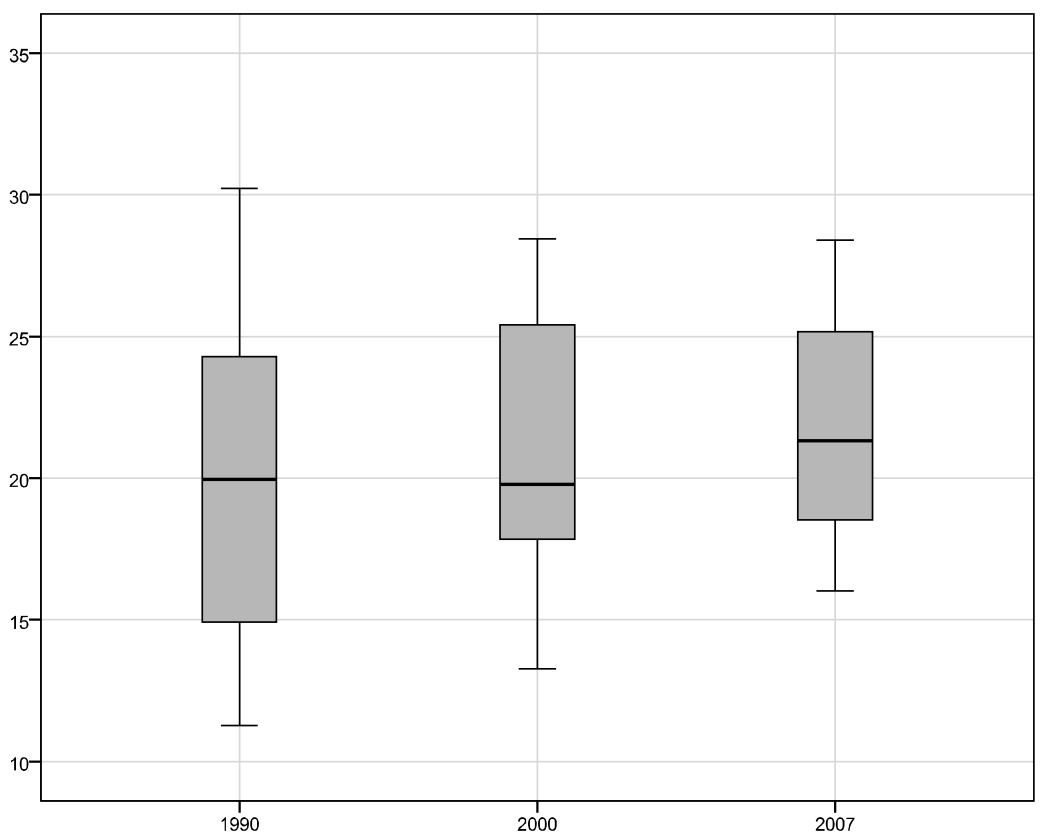

Datenbasis: OECD 2010 b.

Der Rückgang der Staatsquote bei gleichzeitigem Anstieg der Sozialleistungsquote impliziert, dass in der Vergangenheit in anderen Politikfeldern ungleich stärker als in der Sozialpolitik gekürzt wurde. ${ }^{30}$ Opfer der ausgabenseitigen Haushaltssanierung waren dieser Studie zufolge in erster Linie die nicht-sozialstaatlichen Politikfelder, die rund die Hälfte der Staatsausgaben ausmachen. Seit den 1980er Jahren waren in besonderem Maße die Bereiche Bildung, wirtschaftliche Angelegenheiten (z.B. Subventionen) und Verteidigung von Ausgabensenkungen betroffen. Schließlich entlasteten niedrigere Zinssätze in den 1990er Jahren den Staatshaushalt überschuldeter Länder. ${ }^{31}$ Abbildung 6 zeigt anhand von Boxplots die Entwicklung der öffentlichen Ausgaben in diesen vier Bereichen. Mit Ausnahme der Bildungsausgaben zeigt sich bei den Aufwendungen für Landesverteidigung, Subventionen und die Bedienung der Staatsschulden eine klare Konvergenz nach unten - ablesbar an einem sinkenden Median und einer Abnahme der Varianz über die Zeit. Der praktisch konstante Median bei den Bildungsausgaben täuscht darüber hinweg, dass zwischen 1995 und 2007 in zwei Drittel aller Länder die öffentlichen Bildungsausgaben in Relation zum BIP gesunken sind. Zudem ist auch im Bildungssektor die Streuung

30 Castles 2007.

31 Ebd. 
der Ausgaben über die Zeit geringer geworden. Während also bei den Sozialausgaben eine klare Konvergenz nach oben festzustellen ist (Abbildung 5), zeigt sich in einer Reihe von nicht-sozialpolitischen Politikfeldern eine Angleichung der Ausgaben nach unten (Abbildung 6).

\section{Abbildung 6: Entwicklung nicht-sozialpolitischer Ausgaben in 21 OECD Ländern} seit ca. 1990 bis zum Vorabend der Finanzkrise

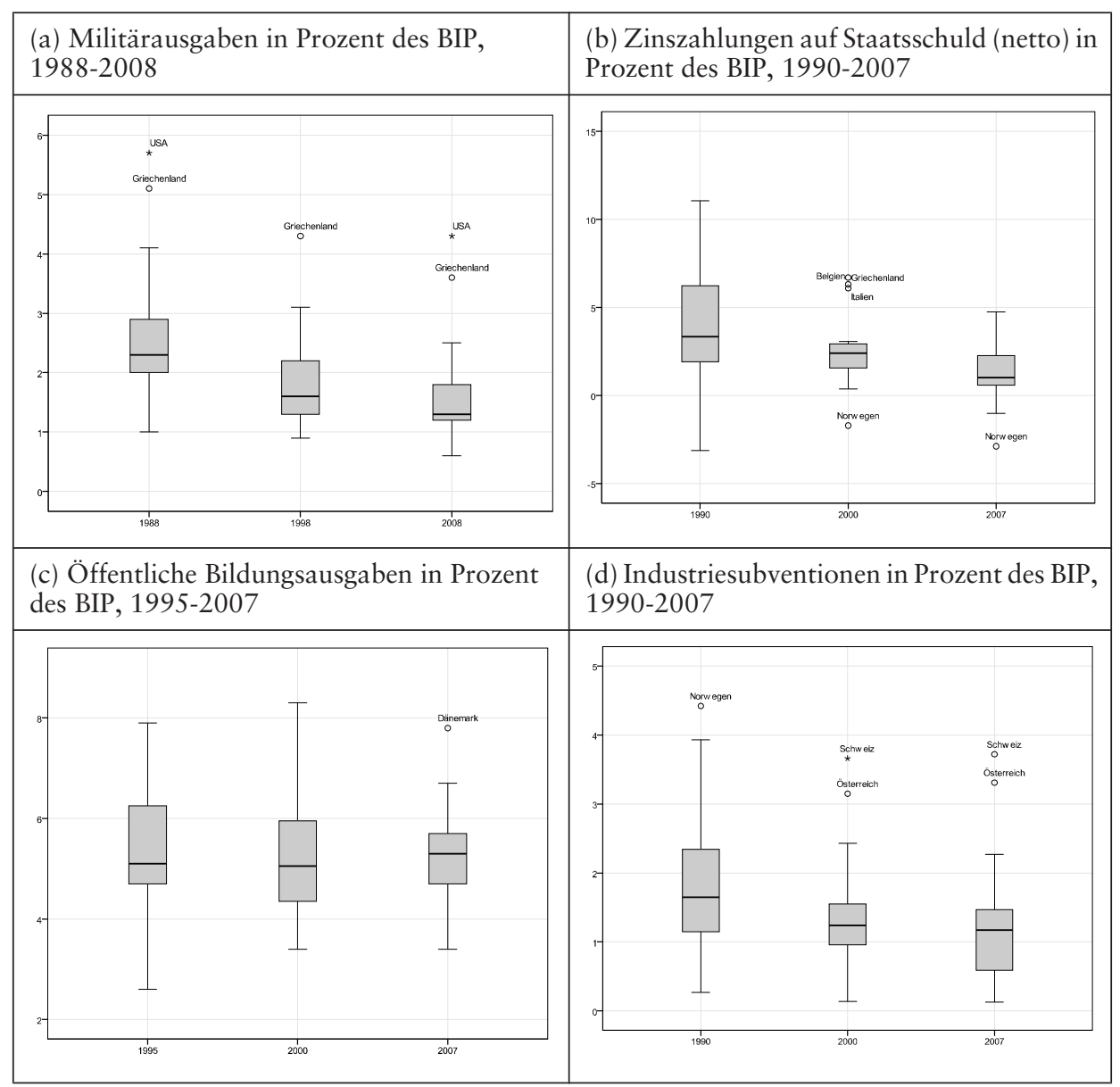

Datenbasis: OECD 2010c, S.243, 2010a; SIPRI Military Expenditure Database (bttp://milexdata.sipri.org/).

Anmerkung: Bildungsausgaben 1995 ohne Belgien.

Paul Piersons Hypothesen liefern für dieses asymmetrische Konsolidierungsmuster eine plausible Erklärung. Aufgrund des hohen politischen Risikos restriktiver Sozialpolitik haben die politischen Eliten den Konsolidierungsdruck offenkundig in demokratisch weniger gut verteidigte Politikfelder umgelenkt. Diese von der Angst 
vor Schuldzuweisungen geleitete Strategie wurde dabei nicht zuletzt durch glückliche Umstände erleichtert. Das Ende des Ost-West-Konflikts ermöglichte beträchtliche Einsparungen bei den Militärausgaben, ${ }^{32}$ während die im Vergleich zu den 1980er Jahren geringeren Zinssätze die Haushalte entlasteten. ${ }^{33}$

Entscheidend für mein Argument ist nun, dass in der Zukunft eine politisch motivierte Umlenkung des Kürzungsdrucks in nicht-sozialpolitische Politikfelder in ähnlicher Größenordnung aus vier Gründen ungleich schwieriger zu bewerkstelligen ist.

Erstens wurden in der Vergangenheit die Ausgaben in einigen Politikfeldern derart stark reduziert, dass weitere Ausgabenkürzungen die Aufgabenerfüllung und Funktionsfähigkeit des Staates in bestimmten Bereichen massiv beeinträchtigen würden. Exemplarisch kann dies anhand der Landesverteidigung illustriert werden: Der Median der Militärausgaben in den 21 OECD-Ländern belief sich im Jahr 2008 auf 1,3 Prozent des BIP (1988: 2,3 Prozent). Sofern man politisch an einer effektiven Landesverteidigung festhalten will, ist eine weitere deutliche Rückführung der Militärausgaben in vielen Ländern wohl kaum noch möglich, nicht zuletzt, weil der rasante Kostenanstieg militärischer Kapitalgüter ${ }^{34}$ umfangreiche Einsparungen im Verteidigungsetat weiter erschwert.

Zweitens machen grundlegend veränderte gesellschaftliche, wirtschaftliche und geopolitische Kontextbedingungen in vielen Politikfeldern eher höhere öffentliche Ausgaben notwendig. Das gilt allen voran für die Bildungsausgaben, da Wissen in postindustriellen Gesellschaften maßgeblich über künftigen Wohlstand und individuelle Aufstiegs- und Teilhabechancen entscheidet. ${ }^{35}$ Hinzu kommen neue terroristische Bedrohungen oder stetig wachsende Herausforderungen in der Klima- und Umweltpolitik.

Drittens handelt es sich bei Politikfeldern wie der Inneren Sicherheit oder der Landesverteidigung um hoheitliche Aufgaben des Staates. Aus verfassungspolitischen Gründen scheidet eine umfangreiche Kostenentlastung des Staates durch Privatisierungen aus. Demgegenüber wäre eine Privatisierung von Bildungsausgaben nach angelsächsischem Muster prinzipiell denkbar, würde aber die Probleme weiter verschärfen, die im letzten Abschnitt diskutiert werden.

Viertens steigen schließlich mit der dramatisch gestiegenen Staatsverschuldung auch die Zinslasten. Während in der Vergangenheit bei diesem Ausgabenposten Einsparungen möglich waren, müssen künftig die Finanzminister in hochverschuldeten Ländern aufgrund höherer Zinsaufschläge wieder größere Beträge in den Haushalt einstellen. Bereits heute sind in Deutschland ca. 15 Prozent des Bundesbudgets für Zinszahlungen reserviert, die damit nach den Sozialausgaben den zweitgrößten Einzeletat repräsentieren. Dies schmälert nicht nur den fiskalischen Handlungsspielraum, sondern erhöht gleichzeitig den Spardruck in anderen Politikfeldern.

32 Cusack 2007.

33 Wagschal, Wenzelburger 2008, S. 61-64.

34 Cusack 2007.

35 Esping-Andersen 2002. 
Zusammenfassend beschränkte sich die strategische Option für die politischen Eliten, den Kürzungsdruck in nicht-sozialstaatliche Bereiche umzulenken, auf ein historisch kurzes Zeitfenster. Dieser Spielraum ist mittlerweile aber weitgehend ausgeschöpft, sodass die sozialen Sicherungssysteme in hochverschuldeten Ländern künftig mit deutlich höherer Wahrscheinlichkeit in das Zentrum von Einsparungen geraten werden. Dies wird jedoch in demokratischen politischen Systemen negative Reaktionen seitens der Wähler provozieren. Der Spardruck wird noch weiter verstärkt, weil auch in der Sozialpolitik im Zuge veränderter gesellschaftlicher und wirtschaftlicher Rahmenbedingungen neue Bedarfslagen und Risiken entstanden sind, deren Bearbeitung eher höhere Sozialausgaben erforderlich machen. Zu denken ist hier beispielsweise an neue soziale Risiken durch die modernisierungsbedingte Pluralisierung von Lebens- und Familienformen, die Alterung von Gesellschaften oder die massive Zunahme atypischer Beschäftigung und damit verbundene Armutsrisiken. ${ }^{36}$ Hinzu kommt schließlich, dass der Wohlfahrtsstaat auch die sozialen Kollateralschäden der Finanz- und Wirtschaftskrise abfedern musste. Dazu gehört nicht nur die Versorgung eines größeren Heers an Arbeitslosen, sondern auch die Einkommensunterstützung für Personen, die im Zuge der Finanzkrise ihre Ersparnisse verloren haben, aus denen sie ihre Altersversicherung bezogen haben. Beispielsweise wurden in den USA Haushaltsvermögen in Höhe von 11 Billionen Dollar vernichtet, darunter auch Pensionsrücklagen und Sparguthaben für die Alterssicherung. ${ }^{37}$

\section{Die Einnahmenseite: alte und neue Restriktionen}

Bislang standen Ausgabenkürzungen im Blickpunkt. Alternativ oder ergänzend kann die Sanierung der öffentlichen Haushalte mit einnahmenseitigen Maßnahmen erfolgen. Ähnlich wie auf der Ausgabenseite haben sich jedoch auch hier die Rahmenbedingungen verändert, sodass neben alten Hindernissen auch neue Restriktionen eine einnahmenseitige Haushaltskonsolidierung künftig erschweren. Paul Piersons Argument gilt zunächst ebenso für die Einnahmenseite. Dass Steuer- und Abgabenerhöhungen unpopulär sind und für wiederwahlorientierte Politiker ein Risiko darstellen, ist alles andere als neu. Was sich aber im Vergleich zu den vorangegangenen Jahrzehnten verändert hat, sind die im Zuge von Europäisierung und Globalisierung entstandenen Möglichkeiten zur Ausschöpfung von internationalen Faktorpreisdifferenzen. Die erleichterten Möglichkeiten zum Standortwechsel haben den Lohnkosten- und Steuerwettbewerb zwischen Nationen deutlich verschärft und neue Restriktionen auf der Einnahmenseite geschaffen. Das gilt sowohl für die Sozialbeiträge als auch die Höhe der Steuersätze. Viele Länder haben Maßnahmen zur Stabilisierung oder Reduzierung der Beitragsbelastung getroffen und die Körperschaftssteuersätze sowie die Spitzensteuersätze bei der Einkommenssteuer gesenkt. Dieser Steuerwettbewerb wurde insbesondere von kleinen Ländern

36 Vgl. Alber 2002.

37 Financial Crisis Inquiry Commission 2011, S. xv.

Leviathan, 40. Jg., 3/2012 
forciert, da dort der Kapitalzustrom aus dem Ausland die durch Steuersenkungen erzeugten Einnahmenausfälle im Inland überkompensiert. ${ }^{38}$ Zwar blieb die Steuerquote relativ stabil, der Redistributionsgrad der Steuersysteme ist allerdings geringer geworden, zumal Lohneinkommen stärker als das mobile Finanzkapital belastet werden. Die Politik steht in Zeiten entgrenzter Märkte vor einem Dilemma. Eine Harmonisierung von Steuersätzen auf europäischer Ebene ist aufgrund heterogener Interessen der Mitgliedsstaaten und hoher Vetoanfälligkeit ebenso wenig in Sicht ${ }^{39}$ wie eine effektive Austrocknung von Steueroasen. Werden vor diesem Hintergrund jedoch die relativ immobilen Faktoren steuerlich stärker belastet, ist zumindest in Hochsteuerländern mit wahlpolitischen Gegenreaktionen seitens der relativ immobilen Lohneinkommensbezieher zu rechnen. ${ }^{40}$ Ein Beispiel sind die Wahlerfolge populistischer Anti-Steuerparteien in einigen skandinavischen Ländern in den 1970er Jahren. An dieser Stelle schließt sich der Kreis zu Paul Piersons Thesen.

Ebenso wie auf der Ausgabenseite fanden die politischen Eliten auf der Suche nach Einnahmen in der Vergangenheit jedoch einen Ausweg. Durch Privatisierungen öffentlicher Unternehmen konnten einmalig hohe Einnahmen zu vergleichsweise geringen politischen Kosten erzielt werden. Allein in den 1990er Jahren wurde weltweit fast eine Billion US-Dollar an Privatisierungserlösen erzielt, mehr als drei Viertel dieser Summe entfielen auf die OECD-Welt. ${ }^{41}$ Dieser Weg ist heute allerdings weitgehend versperrt, weil sich der Staat in der Vergangenheit fast überall in erheblichem Umfang von Unternehmensbeteiligungen getrennt hat (Abbildung 7). Öffentliche Beteiligungen an Industrieunternehmen sind heute eher die Ausnahme als die Regel, und selbst die Daseinsvorsorgebereiche waren, wenngleich in unterschiedlichem Ausmaß, von materiellen Privatisierungen betroffen. ${ }^{42}$ Aufgrund des bereits weitgehend erfolgten "Ausverkaufs des Tafelsilbers « ${ }^{43}$ kommen Privatisierungserlöse daher künftig nur noch in geringem Umfang als Instrument zur Haushaltsentlastung in Frage.

38 Ganghof, Genschel 2008.

39 Scharpf 2008.

40 Scharpf $2000 \mathrm{~b}$.

41 OECD 2003, S. 7.

42 Schmitt 2010.

43 Zohlnhöfer, Obinger 2005. 
Abbildung 7: Umsatzerlöse öffentlicher Unternehmen am BIP in 21 OECD-Ländern, 1980-2007

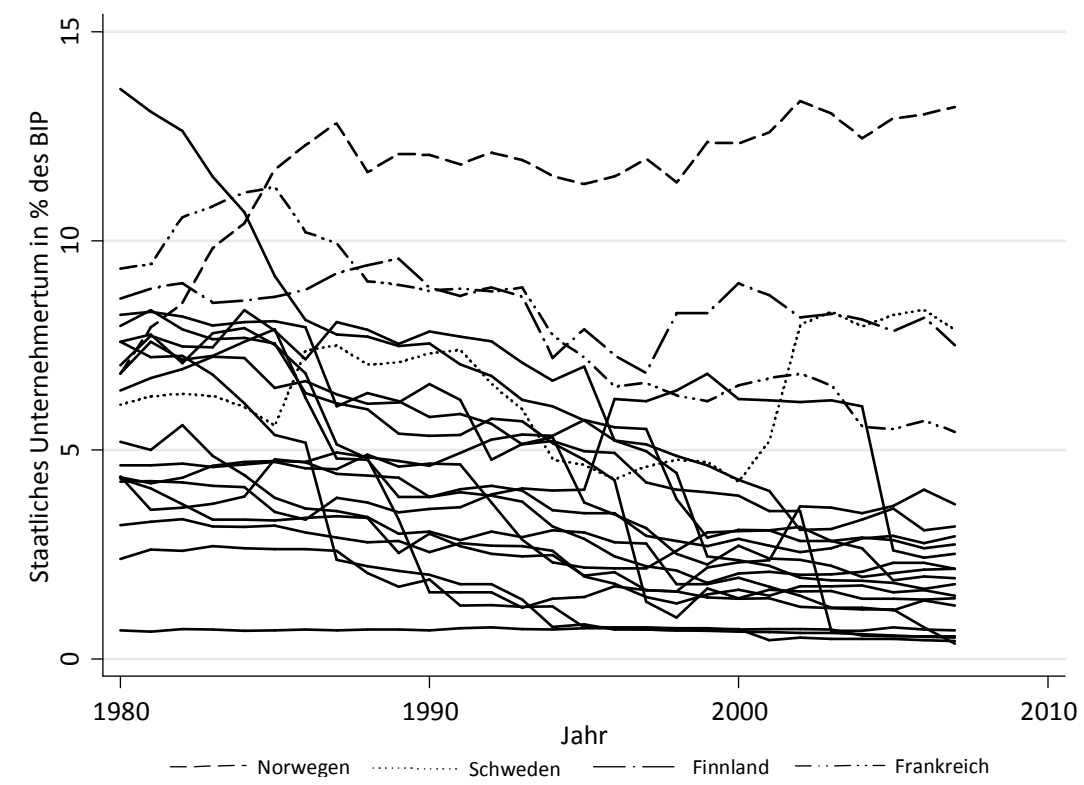

Anmerkung: Aus Gründen der Übersichtlichkeit sind nur jene Länder hervorgehoben, die vom allgemeinen Trend abweichen.

Quelle: Eigene Erhebungen im Rabmen des Projekts »Der Rückzug des Staates aus unternehmerischen Tätigkeiten«, Sfb 597, Universität Bremen. Für Details zur Messung vgl. Schmitt, Schuster 2010.

\section{Folgen und Ausblick}

In Reaktion auf den Anstieg der Staatsverschuldung seit den 1970er Jahren wurden die öffentlichen Haushalte in der Vergangenheit maßgeblich durch geräuscharme Ausgabenkürzungen in den nicht-sozialstaatlichen Politikfeldern sowie mit Einmalmaßnahmen auf der Einnahmenseite konsolidiert. Aufgrund wahlstrategischer Motive der politischen Eliten kam der Wohlfahrtsstaat dabei relativ ungeschoren davon. Umbau und nicht umfassender Rückbau prägte die Sozialpolitik der letzten drei Jahrzehnte. Die Ausgaben für Sozialpolitik sind seit 1980 aufgrund steigender Arbeitslosigkeit, demografischer Veränderungen und höherer Gesundheitskosten sogar beträchtlich angestiegen ${ }^{44}$ und absorbieren heute fast überall mehr als die Hälfte aller öffentlichen Ausgaben.

Im Zuge der jüngsten Finanz- und Wirtschaftskrise ist in fast allen reichen Demokratien die Staatsverschuldung durch Übernahme privater Schulden systemrelevan-

44 Nochmals soll betont werden, dass dieser Ausgabenanstieg Leistungskürzungen nicht ausschließt. 
ter Unternehmen und Banken und durch Konjunkturprogramme explodiert, sodass Entschuldung, Haushaltssanierung und Haushaltsdisziplin für längere Zeit die politische Agenda bestimmen werden. Da sich wesentliche Konsolidierungsoptionen der Vergangenheit, nämlich Ausgabenkürzungen in nicht-sozialstaatlichen Politikfeldern sowie Privatisierungen, mittlerweile in hohem Maße erschöpft haben, ist der New Politics of the Welfare State gewissermaßen die Geschäftsgrundlage abhanden gekommen. Zudem sind Steuer- und Beitragserhöhungen heute wohl schwerer durchzusetzen als jemals zuvor. ${ }^{45}$ Vor dem Hintergrund des großen fiskalischen Gewichts des Sozialstaates und des Umstands, dass andere Politikfelder nicht mehr als Blitzableiter für Ausgabenkürzungen fungieren können, sehen sich die politischen Eliten angesichts einer prekären Haushaltslage verstärkt gezwungen, unpopuläre Entscheidungen in der Sozialpolitik zu implementieren. Dies gilt selbst für einen Konsolidierungskurs, der (realistischerweise) Ausgabenkürzungen mit Einnahmensteigerungen kombiniert. Wie intensiv diese Einschnitte ausfallen, variiert jedoch stark mit dem fiskalischen Problemdruck. Die im Jahr 2011 von Ländern wie Irland, Großbritannien, Portugal und Griechenland aufgelegten Sparpakete beinhalten zum Teil drakonische Einschnitte im Sozialbereich und liefern erste Belege für die empirische Tragfähigkeit der Thesen dieses Beitrags. Demgegenüber fielen in Staaten mit geringerem Schuldendruck die Einsparungsmaßnahmen bislang deutlich moderater aus.

Wenn nun die Strategien zur Abwehr von Schuldzuweisungen nicht mehr zur Verfügung stehen und die Politik nicht zuletzt aufgrund des Drucks der Finanzmärkte einen harten und bei den Wählern unpopulären Austeritätskurs durchsetzen soll, der nun zwangsläufig auch den Sozialetat mit einschließt, dann ist künftig nicht nur mit einem höheren Konfliktniveau ${ }^{46}$, sondern auch mit negativen Rückkopplungseffekten auf Politik und Demokratie zu rechnen. Besonders deutlich ist dies bereits in Griechenland zu beobachten, wo externer Druck die Regierung auf einen harten Sparkurs verpflichtet, der unter dem Damoklesschwert des Staatsbankrotts gegen massive Proteste der Bürger durchgesetzt werden muss.

Da der Auf- und Ausbau des Wohlfahrtsstaates in der Nachkriegszeit erfolgreich zur Eindämmung sozialer Ungleichheit beigetragen hat, ${ }^{47}$ wird seine Zurückdrängung die soziale Ungleichheit erhöhen und damit einen Trend der letzten zwei Jahrzehnte weiter fortschreiben. ${ }^{48}$ Gleichzeitig befördert die Fiskalkrise mittel- und langfristig jene Reformtendenzen, die bereits in den letzten zwei Jahrzehnten die sozialstaatliche Umbauagenda geprägt haben, nämlich Privatisierung von Sozialaufgaben, Neugestaltung der Anreizsysteme und Aktivierung. ${ }^{49}$ (Teil-)Privatisie-

45 Streeck, Mertens 2010, S. 24.

46 Hauptkonfliktpunkt ist die Frage, wer die Konsolidierungslasten trägt. Dies betrifft sowohl die nationale als auch die internationale (europäische) Ebene. Die Debatte um eine europäische Transfer- bzw. Schuldenunion belegt dies mehr als deutlich.

47 Brady 2009.

48 OECD 2008.

49 Gilbert 2004. 
rungen sozialer Sicherungssysteme (z.B. der Alterssicherung) verstärken jedoch soziale Segmentierungsprozesse bzw. die Dualisierung zwischen In- und Outsidern..$^{50}$

Sozialstaatskürzungen und ein Anstieg sozialer Ungleichheit bleiben aber nicht folgenlos für Politik und Demokratie, da der Wohlfahrtsstaat in der Vergangenheit wichtige politische Integrationsleistungen erbracht hat. ${ }^{51}$ Versteht man soziale Rechte im Sinne von T.H. Marshall als Teilhaberechte und -chancen und teilt man die Einschätzung, dass der Wohlfahrtsstaat zur Stabilisierung und Legitimität der Demokratie beigetragen hat, dann sind im Hinblick auf die politischen Folgewirkungen von Sozialstaatsrückbau drei Aspekte von Relevanz.

Erstens stellt sich die Frage nach den Auswirkungen wachsender Ungleichheit auf die politische Partizipation der Reformverlierer. Hier sind vielfältige Reaktionsmuster denkbar, die von einer Radikalisierung bis hin zu politischer Apathie reichen, wie es das Beispiel Griechenland bereits zeigt. ${ }^{52}$ Die wenigen empirischen Studien deuten auf einen politischen Entfremdungseffekt bei den Reformverlierern sowie auf die Zunahme unkonventioneller Partizipationsformen von Personen mit hohem Einkommen und Bildungsstand hin. ${ }^{53}$ Wenn dies zutrifft, dann werden soziale Spaltungen durch politische Segmentierungsprozesse gespiegelt, und die Demokratie verkommt mitunter zu einem mit Showelementen angereicherten Elitenschauspiel. $^{54}$

Zweitens liegt die Vermutung nahe, dass Wohlfahrtsstaatsrückbau nicht folgenlos für den Parteienwettbewerb bleibt. Wenn künftig auch die klassischen Sozialstaatsparteien zu unpopulären und schmerzhaften Maßnahmen in der Sozialpolitik gezwungen sind, dann ist seitens ihrer Kernwähler mit massiven Protestreaktionen zu rechnen, von denen insbesondere die Ränder des politischen Spektrums profitieren werden. Die unmittelbare Folge wäre eine größere politische Instabilität und eine noch höhere Kurzatmigkeit der Politik - allesamt Voraussetzungen, die die Handlungsfähigkeit des Staates verringern und damit die Chancen einer nachhaltigen Budgetkonsolidierung schmälern.

Drittens beraubt eine Zurückdrängung des Wohlfahrtsstaates die Demokratie einer wichtigen Quelle von Outputlegitimität. ${ }^{55}$ Wenn die öffentlichen Haushalte durch Ausgabenkürzungen im Sozialbereich konsolidiert werden, gleichzeitig aber aus Gründen der »Standortlogik «56 auf einnahmenseitige Maßnahmen bei den Vermögenden und Besserverdienenden verzichtet wird, dann erschüttert dies grundlegende Fairnessvorstellungen der Bürger und ihr Vertrauen in Demokratie und

50 Palier 2010.

51 Schmidt 2005, S. $287 \mathrm{ff}$.

52 Vgl. Keller 2011.

53 Solt 2008; Schäfer 2009, 2010.

54 Crouch 2008.

55 Scharpf 2000 a, S. 255.

56 Hassel, Lütz 2010, S. 253. 
Marktwirtschaft. Die »Demokratie im Defizit « 57 führt mitunter zu einem veritablen Demokratiedefizit. Die Schuldenkrise schadet damit langfristig nicht nur dem Wohlfahrtsstaat, sondern in weiterer Folge auch der Demokratie. ${ }^{58}$ Sie stellt schließlich auch den Staat auf lange Sicht auf eine harte Belastungsprobe, wenn sie ihn nicht wie das griechische Beispiel zeigt - sogar überfordern wird. ${ }^{59}$

Nochmals muss allerdings betont werden, dass der fiskalische Problemdruck sich nicht überall in ähnlicher Schärfe stellt und zentrale makroökonomische Schlüsselgrößen wie das Wirtschaftswachstum analytisch bislang ausgeklammert blieben. Tatsächlich handelt es sich dabei um eine zentrale Unbekannte, deren langfristige Prognose kaum seriös möglich ist. Eine länger andauernde günstige Konjunkturphase kann die Haushaltskonsolidierung wesentlich erleichtern. Für diesen Optimismus besteht trotz vorübergehend hoher Wachstumsraten aber wenig Anlass, weil fast alle entwickelten Demokratien mittelfristig einem Sparzwang unterliegen, der das kurzfristig hohe und maßgeblich durch Aufholeffekte getragene Wachstum der unmittelbaren Nachkrisenzeit konterkariert. Zudem ist die Krisengefahr noch nicht gebannt. Eine weitere Finanzkrise hätte wohl fatale Konsequenzen. ${ }^{60}$ All das ist jedoch Spekulation. Sicher ist hingegen, dass die Schuldenkrise nicht unbegrenzt andauern kann. Ihre Eindämmung wird jedenfalls (sozial)politisch turbulent.

\section{Literatur}

Adema, Willem; Fon, Pauline; Ladaique, Maxime 2011. Is the European welfare state really more expensive? OECD social, employment and migration working papers No. 124. Paris: OECD.

Alber, Jens 2002. »Modernisierung als Peripetie des Sozialstaats? «, in Berliner Journal für Soziologie 12, S. 5-35.

Brady, David 2009. Rich democracies, poor people. How politics explain poverty. New York, Oxford: Oxford University Press.

Castles, Francis G. Hrsg. 2007. The disappearing state? Retrenchment realities in an age of globalisation. Cheltenham: Edward Elgar.

Crouch, Colin 2008: Postdemokratie. Frankurt a. M. Suhrkamp.

Cusack, Thomas R. 2007. "Sinking budgets and ballooning prices: recent developments connected to military spending ", in The disappearing state? Retrenchment realities in an age of globalisation, hrsg. v. Castles, Francis G., S. 103-132, Cheltenham: Edward Elgar.

Esping-Andersen, Gøsta 2002. Why we need a new welfare state. Oxford: Oxford University Press.

Financial Crisis Inquiry Commission 2011: The financial crisis inquiry report. Final report of the national commission on the causes of the financial and economic crisis in the United States. Washington D.C.

Ganghof, Steffen; Genschel, Philipp 2008. »Deregulierte Steuerpolitik: Körperschaftsteuerwettbewerb und Einkommensbesteuerung in Europa «, in Politische Ökonomie der Europäischen Integration, hrsg. Höpner; Martin; Schäfer, Armin, S. 311-333. Frankfurt a. M.: Campus.

Gilbert, Neil 2004. Transformation of the welfare state. The silent surrender of public responsibility. Oxford: Oxford University Press.

57 Streeck, Mertens 2010, S. 23.

58 Schäfer 2010.

59 Klenk, Nullmeier 2010, S. 289-290.

60 Streeck 2010. 
Hassel, Anke; Lütz, Susanne 2010. »Durch die Krise aus der Krise? Die neue Stärke des Staates «, in der moderne staat 3, S. 251-271.

Hemerijck, Anton 2011. 21st century welfare provision is more than the social insurance statea reply to Paul Pierson. ZeS-Arbeitspapier 3/2011, Zentrum für Sozialpolitik, Universität Bremen.

Kaasch, Alexandra; Starke, Peter; Hooren, Franca van 2010. The welfare state as crisis manager? Comparing social policy responses to three major economic crises. Paper presented at the APSA's annual meeting, Washington, D.C., September 2-5, 2010.

Keller, Carsten 2011. »Ungleichheit und Konflikt: zur Relevanz staatlicher Legitimation in mikrosozialen Statuskämpfen «, in Leviathan 39, 4, S. 567-582.

Klenk, Tanja; Nullmeier, Frank 2010. »Politische Krisentheorien und die Renaissance von Konjunkturprogrammen ", in der moderne staat 3, S. 273-294.

Korpi, Walter; Palme, Joakim 2003. »New politics and class politics in the context of austerity and globalization: welfare state regress in 18 countries, 1975-95 ", in American Political Science Review 97, S. 425-446.

OECD 2003. Privatising state-owned enterprises. An overview of policies and practices in OECD countries. Paris: OECD.

OECD 2008. Growing unequal? Income distribution and poverty in OECD countries. Paris: OECD.

OECD 2010 a. OECD economic outlook No. 88 (database). http://stats.oecd.org/Index.aspx? QueryId=26622 (Zugriff vom 16.03.2011).

OECD 2010 b. Social expenditure database. Paris: OECD.

OECD 2010 c. Education at a glance. Paris: OECD.

OECD 2011. OECD economic outlook No. 90. Paris: OECD.

Palier, Bruno. Hrsg. 2010. A long goodbye to Bismarck? The politics of welfare reform in continental Europe. Amsterdam: Amsterdam University Press.

Pierson, Paul 1994. Dismantling the welfare state? Reagan, Thatcher, and the politics of retrenchment. Cambridge: Cambridge University Press.

Pierson, Paul 1996. " The new politics of the welfare state «, in World Politics 48, S. 143-179.

Pierson, Paul 2011. The welfare state in the very long-run. ZeS-Arbeitspapier 2/2011, Zentrum für Sozialpolitik, Universität Bremen.

Reinhart, Carmen M.; Rogoff, Kenneth S. 2009. This time is different. Eight centuries of financial folly. Princeton: Princeton University Press.

Schäfer, Armin 2009. "Krisentheorien der Demokratie: Unregierbarkeit, Spätkapitalismus und Postdemokratie ", in der moderne Staat 2, S. 159-183.

Schäfer, Armin 2010. »Die Folgen sozialer Ungleichheit für die Demokratie in Westeuropa «, in Zeitschrift für Vergleichende Politikwissenschaft 4, S. 131-156.

Scharpf, Fritz W. 2000 a. Interaktionsformen. Akteurzentrierter Institutionalismus in der Politikforschung. Wiesbaden: VS Verlag für Sozialwissenschaften.

Scharpf, Fritz W. 2000 b. »The viability of advanced welfare states in the international economy: vulnerabilities and options ", in Journal of European Public Policy 7, S. 190-228.

Scharpf, Fritz W. 2008. "Negative und positive Integration (inklusive Nachbetrachtung) «, in Politische Ökonomie der Europäischen Integration, hrsg. v. Höpner; Martin; Schäfer, Armin, S. 49-99. Frankfurt a. M.: Campus.

Schmidt, Manfred G. 2005. Sozialpolitik in Deutschland. Historische Entwicklung und internationaler Vergleich. Wiesbaden: VS Verlag für Sozialwissenschaften.

Schmitt, Carina 2010. »Der Staat als Unternehmer bei der Bereitstellung öffentlicher Dienstleistungen - Konvergenz im internationalen Vergleich? ", in der moderne staat 3, S. 455-469.

Schmitt, Carina; Schuster, Philipp 2010. New public management reform in European countries: the retreat of the state from telecommunication services. TranState working papers 116/2010. Universität Bremen.

Solt, Frederick 2008. "Economic inequality and democratic political engagement ", in American Journal of Political Science 52, S. 48-60.

Streeck, Wolfgang 2009. Re-forming capitalism. Institutional change in the German political economy. Oxford: Oxford University Press.

Streeck, Wolfgang 2010. »Noch so ein Sieg, und wir sind verloren. Der Nationalstaat nach der Finanzkrise", in Leviathan 38, S. 159-173.

Leviathan, 40. Jg., 3/2012 
Streeck, Wolfgang; Mertens, Daniel 2010. »Politik im Defizit: Austerität als fiskalpolitisches Regime", in der moderne staat 3, S. 7-29.

Svallfors, Stefan 2010. »Public attitudes ", in The Oxford handbook of the welfare state, hrsg. v. Castles, Francis G. et al., S. 241-251. Oxford: Oxford University Press.

Vis, Barbara; Kersbergen, Kess van; Hylands, Tom 2011. "To what extent did the financial crisis intensify the pressure to reform the welfare state? ", in Social Policy \& Administration 45, S. 338-353.

Wagschal, Uwe; Jäkel, Tim 2010. »Öffentliche Finanzen im Stresstest - Policy-Reaktionen auf die Finanz- und Wirtschaftskrise", in der moderne staat 3, S. 295-320.

Wagschal, Uwe; Wenzelburger, Georg 2008. Haushaltskonsolidierung. Wiesbaden: VS Verlag für Sozialwissenschaften.

Wenzelburger, Georg 2010. Haushaltskonsolidierungen und Reformprozesse. Determinanten, Konsolidierungsprofile und Reformstrategien in der Analyse. Münster: LIT Verlag.

Zohlnhöfer, Reimut 2009: Globalisierung der Wirtschaft und finanzpolitische Anpassungsreaktionen in Westeuropa. Baden-Baden: Nomos.

Zohlnhöfer, Reimut; Obinger, Herbert 2005. »Der Ausverkauf des >Tafelsilbers ‘: Bestimmungsfaktoren der Privatisierungserlöse in EU- und OECD-Staaten 1990-2000 «, in Politische Vierteljahresschrift 46, S. 602-628. 
Zusammenfassung: Dieser Beitrag beschäftigt sich mit der Zukunft des Wohlfahrtsstaates nach der weltweiten Finanzkrise. Es wird argumentiert, dass es aufgrund der jüngsten Rezession in den kommenden Jahren zu erheblichen Einschnitten im Sozialbereich kommen wird. Antriebskräfte dafür sind (a) die durch den Wirtschaftseinbruch verursachte Fiskalkrise, (b) das große fiskalische Gewicht reifer Wohlfahrtsstaaten, (c) die Ausschöpfung von Kürzungen in einer Reihe von nicht-sozialstaatlichen Politikfeldern und (d) Restriktionen auf der Einnahmenseite aufgrund von Globalisierung und Europäisierung. Die erwartete Zurückdrängung des Sozialstaates wird sich negativ auf Politik und Demokratie auswirken.

Stichworte: Finanzkrise, Wohlfahrtsstaat, Staatsschulden

\section{The financial crisis and the future of the welfare state}

Summary: This article speculates about the future of the welfare state following the global financial crisis. It argues that the recent recession will cause marked welfare state retrenchment in the years to come. Retrenchment is motivated by (a) the recent economic downturn and the resulting fiscal crisis, (b) the significant fiscal weight of mature welfare states, (c) the exhaustion of budgetary cutbacks in a number of policy areas beyond social security, and (d) the restrictions imposed by globalization and Europeanization on public revenues. The expected welfare cutbacks will have negative consequences for democratic policies.

Keywords: Financial crisis, welfare state, public debt

Autor

Prof. Dr. Herbert Obinger

Zentrum für Sozialpolitik

Universität Bremen

Mary-Somerville-Straße 5

28359 Bremen

hobinger@zes.uni-bremen.de

Leviathan, 40. Jg., 3/2012 https://doi.org/10.5771/0340-0425-2012-3-441 